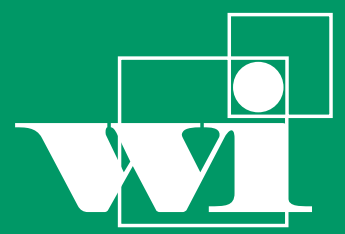

Bayreuther Arbeitspapiere zur Wirtschaftsinformatik

Torsten O. Paulussen, Franz Rothlauf, Armin Heinzl

Konzeption eines Koordinationsmechanismus zur dezentralen Ablaufplanung in medizinischen Behandlungspfaden (MEDPACO)

Bayreuth Reports on Information Systems Management 
Die Arbeitspapiere des Lehrstuhls für Wirtschaftsinformatik dienen der Darstellung vorläufiger Ergebnisse, die i. d. R. noch für spätere Veröffentlichungen überarbeitet werden. Die Autoren sind deshalb für kritische Hinweise dankbar.

Alle Rechte vorbehalten. Insbesondere die der Übersetzung, des Nachdruckes, des Vortrags, der Entnahme von Abbildungen und Tabellen auch bei nur auszugsweiser Verwertung.

\section{Authors:}

Torsten O. Paulussen

Franz Rothlauf

Armin Heinzl
The Bayreuth Reports on Information Systems Management comprise preliminary results which will usually be revised for subsequent publications. Critical comments would be appreciated by the authors.

All rights reserved. No part of this report may be reproduced by any means, or translated.

\section{Information Systems Management Working Paper Series}

\section{Edited by:}

Prof. Dr. Armin Heinzl

\section{Managing Assistant and Contact:}

Universität Bayreuth

Lehrstuhl für Wirtschaftsinformatik (BWL VII)

Prof. Dr. Torsten Eymann

Universitätsstrasse 30

95447 Bayreuth

Germany

Email: wi@uni-bayreuth.de
ISSN $1864-9300$ 


\title{
Konzeption eines Koordinationsmechanismus zur dezentralen Ablaufplanung in medizinischen Behandlungspfaden (MEDPACO)
}

\author{
Torsten O. Paulussen Franz Rothlauf Armin Heinzl
}

Lehrstuhl BWL VII

Universität Bayreuth

Universitätsstraße 30, D-95440 Bayreuth

paulussen,rothlauf,heinzl@uni-bayreuth.de 


\title{
Konzeption eines Koordinationsmechanismus zur dezentralen Ablaufplanung in medizinischen Behandlungspfaden (MEDPACO)
}

\begin{abstract}
Zusammenfassung:
In der vorliegende Arbeit wird eine Konzeption für ein Koordinationsverfahren zur dezentralen Ablaufplanung in medizinischen Behandlungspfaden (MedPaCo) entwickelt. Hierzu werden die ablaufplanerisch relevanten Eigenschaften von Krankenhausprozessen analysiert und ein Überblick über die wesentlichen Beiträge zur dezentralen Planung in Krankenhäusern gegeben. Im Anschluß daran wird die Architektur von MedPaCo beschrieben. Basierend auf einem Multiagentensystem greift MedPaCo das Konzept der marktwirtschaftichen Koordination auf. Hierbei werden die Patienten und Funktionsbereiche im Krankenhaus durch Patienten- und Ressourcenagenten repräsentiert, die über eigene Ziele und Restriktionen verfügen. Danach wird der Koordinationsablauf dargestellt und an einem Beispiel illustriert. Zum Schluß wird eine erste Würdigung von MedPaCo vorgenommen und auf offene Probleme eingegangen.
\end{abstract}

Schlüsselworte: Patientensteuerung, Koordination, dezentrale Ablaufplanung, marktanaloge Koordination, Multiagentensysteme 


\section{Einleitung}

Der vorliegende Beitrag beschäftigt sich mit der Konzeption eines Koordinationsmechanismus zur dezentralen Ablaufplanung in medizinischen Behandlungspfaden.

Krankenhäuser bestehen aus einer Vielzahl von dezentral organisierten Funktionsbereichen, wie z.B. der Radiologie, dem Operationsbereich oder der Bettenstation. Die Patienten durchlaufen während ihres Krankenhausaufenthalts - in Abhängigkeit von ihrer Erkrankung - die für sie relevanten Funktionsbereiche.

Hierbei verfolgen die einzelnen Patienten und Ressourcen Zielsetzungen, die in einem konkurrierenden Verhältnis zueinander stehen können (vgl. [DeLi99, S. 3; Gäf90]). Ein Beispiel hierfür ist die Reduzierung von Leerlaufzeiten und die Erreichung einer möglichst kurzen Durchlaufzeit. Dadurch wird die Patientensteuerung mit dem Dilemma der Ablaufplanung konfrontiert [Adam93, S. 37 ff.].

Die Aufgabe der Patientensteuerung ist es nun, die Patienten in der Weise in die jeweiligen Funktionsbereiche einzuplanen, daß sowohl die Ziele der Patienten, als auch der Funktionsbereiche möglichst gut erfüllt werden. Aufgrund des dezentralen Charakters der Krankenhausorganisation und der damit einhergehenden dezentralen Informationsverteilung, bietet sich ein dezentraler Koordinationsmechanismus zur Abstimmung der einzelnen Pläne und Ziele an.

Da es derzeit nur wenige Ansätze ([DeLi98; SchmUr99]) zur dezentralen Steuerung von Krankenhausprozessen gibt, soll diese Arbeit einen ersten Beitrag zur Weiterentwicklung von dezentralen Einplanungsverfahren in Krankenhausprozessen leisten.

Nach einer kurzen Beschreibung der für die Patientensteuerung relevanten Eigenschaften von Krankenhausprozessen wird ein kurzer Überblick über die wesentlichen Arbeiten zur dezentralen Planung und Koordination von Krankenhausprozessen gegeben. Im Anschluß daran wird mit MedPaCo eine eigene Konzeption eines Koordinationsmechanismus zur dezentralen Ablaufplanung erarbeitet und diskutiert. Zum Schluß wird ein Fazit gezogen und ein Ausblick auf weitere Entwicklungen gegeben.

\section{Krankenhausprozesse}

Der Behandlungsprozeß eines Patienten im Krankenhaus setzt sich in der Regel aus mehreren Behandlungen und Untersuchungen zusammen, die in 
unterschiedlichen Funktionsbereichen erbracht werden. Welche Untersuchungen und Behandlungen am Patienten durchgeführt werden müssen, wird durch medizinische Kriterien determiniert (vgl. [Schl90, S. 25]).

Die im Krankenhaus zu erbringenden Leistungen lassen sich in patientengebundene und patientenungebundene Leistungen unterteilen (vgl. [Schl90, S. 15 f.]). Patientengebundene Leistungen sind Behandlungen oder Untersuchungen, bei denen die Anwesenheit des Patienten erforderlich ist, also die Produktion und Konsumption der Leistung gemäß dem uno-actu-Prinzip zusammenfallen [Her94, S.633]. So ist zum Beispiel die Anwesenheit des Patienten zur Erstellung einer Röntgenaufnahme zwingend erforderlich. Dadurch wird der Patient zu einer exklusiven Ressource (vgl. [DeLi99, S. 2]). Im Gegensatz dazu ist die Anwesenheit des Patienten beispielsweise bei einer Blutuntersuchung im Labor oder bei der Auswertung (Befundung) von Röntgenaufnahmen nicht notwendig. In diesem Fall kann von patientenungebundenen Leistungen gesprochen werden. Während patientengebundene Leistungen nur sequentiell erfolgen können, sind patientenungebundene Leistungen prinzipiell parallelisierbar. Im Krankenhaus dominieren die patientengebundenen Leistungen, wodurch der Dienstleistungscharakter von Krankenhausleistungen deutlich wird.

Zwischen den einzelnen Leistungen müssen jedoch keine sequentiellen Interdependenzen bestehen, so daß die Reihenfolge der Behandlungen eines Patienten nicht durch medizinische Restriktionen in allen Teilen fest vorgegeben ist [Gie76, S. 204 f.]. Darüber hinaus besteht bei einigen Patienten das Problem, daß die durchzuführenden Maßnahmen nicht a priori, d.h. bei Ankunft des Patienten im Krankenhaus, feststehen. In diesem Fall kann das Informationsdefizit über die für den Patienten durchzuführenden Behandlungen nur schrittweise reduziert werden [Wen87].

\section{Stand der Forschung}

Die einzelnen Funktionsbereiche sind im Krankenhaus in der Regel dezentral organisiert [DeLi99]. Die für einen Patienten durchzuführenden Behandlungen werden von einem Arzt auf der jeweiligen Station festgelegt und in Form von Anforderungen an die jeweiligen Funktionsbereiche weitergeleitet. Die Funktionsbereiche rufen darauf hin - in Abhängigkeit von ihrer Auslastung - die Patienten von der jeweiligen Station ab. Hierbei findet jedoch keine Koordination zwischen den einzelnen Funktionsbereichen statt, wodurch es zu Wartezeiten für die Patienten und Leerlaufzeiten für die Funktionsbereiche kommen kann.

Aufgrund der hohen Dynamik und Komplexität von Krankenhausprozessen soll im folgenden ein dezentraler Planungsansatz gewählt werden. Eine dezentrale Planung bietet den Vorteil, daß die ablaufplanerisch relevanten Informationen im Sinne von Zielen, Präferenzen und Restriktionen der Beteiligten - nicht bei 
einem zentralen Planer vorliegen müssen, sondern dezentral bei den jeweiligen Beteiligten verbleiben können [Adam93, S. 471]. Hierdurch wird die Möglichkeit geboten, Veränderungen im Entscheidungsfeld sowie Expertenwissen zu erfassen und eine Koordination ohne Überlastung der Entscheidungsinstanz durchzuführen [Adam96].

Zur dezentralen Planung in Krankenhäusern sind den Autoren bisher nur agentenbasierte Ansätze bekannt. Maßgeblich existieren hierzu folgende Beiträge:

- [DeLi98] entwickelten ein System zur Einplanung von Patienten im Krankenhaus auf der Basis eines Multiagentensystems. Zur Abstimmung bei Ressourcenkonflikten (z.B.: ein Patient wird gleichzeitig von verschieden Funktionsabteilungen angefordert) entwickeln sie einen auf dem "Generalized Partial Global Planning (GPGP)" basierenden Koordinationsmechanismus, der im Auktionsverfahren demjenigen Funktionsbereich den Patienten zuteilt, der aufgrund seiner Ressourcenauslastung das höchste Gebot abgibt. Behandlungen, die eine hohe Anzahl von Folgebehandlungen aufweisen, bekommen bei diesem Modell eine höhere Priorität als Behandlungen bei denen die Anzahl der weiteren Untersuchungen klein ist. Ebenso bekommt eine Behandlung, die früher starten kann als eine andere, eine höhere Priorität. Wie diese beiden unterschiedlichen Prioritäten zu einer Größe zusammengefaßt werden, wird jedoch nicht weiter ausgeführt (vgl. auch [DeLi99]).

- Dem Ansatz PECS (Physis, Emotion, Cognition, (Perception), Sensor) von [SchmUr99] liegen Agenten $\mathrm{zu}$ Grunde, die versuchen, physische Eigenschaften, emotionale Zustände, kognitive Fähigkeiten und soziale Merkmale von Menschen abzubilden. Zur Einplanung der Patienten werden sowohl Ressourcen (Personal, Räume, Geräte) als auch Patienten als Agenten modelliert. Durch eine hierarchische Abbildung der Funktionsbereiche ist es möglich, diese zunächst als Ganzes zu modellieren und dann in ihre einzelnen Bestandteile zu unterteilen. Die Patienten-Agenten erhalten anschließend eine Liste mit den zu durchlaufenden Funktionsbereichen. Patientenungebundene Aktivitäten werden bisher nicht berücksichtigt. Auf welcher Basis die Koordination der Agenten stattfindet, ist ebenfalls nicht ersichtlich. Gleiches gilt für die Modellierung der sozio-emotionalen Merkmale. Zudem sollen die Verhandlungen aller Agenten über eine zentrale Softwarekomponente erfolgen. Da sich das System noch in der Konzeptionsphase befindet, können noch keine Aussagen gemacht werden, wie sich dieses Element bei hohen Transaktionsraten verhält.

Dezentrale Planungsansätze in Form von Multiagentensystemen befinden sich am Anfang ihrer Entwicklung. Bislang beschäftigen sich wenige Ansätze mit der Ablaufplanung in Krankenhäusern. Es fällt auf, daß eine starke Betonung der Ressourcen (i. Ggs. zu Patienten) stattfindet. Die zur dezentralen Abstimmung erforderlichen Koordinationsmechanismen werden allenfalls rudimentär oder gar 
nicht systematisiert. Diese Defizite sollen nachfolgend aufgegriffen und vermindert werden.

\section{Konzeption von MedPaCo}

Im folgenden soll mit MedPaCo (Medical Path Coordinator) eine eigene Konzeption eines Koordinationsmechanismus zur dezentralen Ablaufplanung für ein Multiagentensystem entwickelt werden. Hierzu werden zunächst die dem Modell zugrundeliegenden Annahmen dargelegt. Danach erfolgt eine kurze Beschreibung der Architektur des Multiagentensystems. Im Anschluß daran wird das eigentliche Koordinationsverfahren beschrieben, an einem Beispiel verdeutlicht und eine erste Würdigung der Konzeption durchgeführt. Zum Schluß werden offene Probleme angesprochen und erste, mögliche Lösungsansätze vorgestellt.

\subsection{Modellannahmen}

Zur Modellierung des Einplanungsverfahrens wird von folgenden Annahmen ausgegangen:

1. Die Kapazitäten der Funktionsbereiche können voneinander abweichen.

2. Zu jedem Zeitpunkt wird jeder Patient höchstens von einem Funktionsbereich behandelt. Jedoch können für diesen Patienten mehrere patientenungebundene Aktivitäten gleichzeitig durchgeführt werden.

3. Die Behandlungsfolge eines Patienten ist nur teilweise fest vorgegeben.

4. Die Funktionsbereiche stehen kontinuierlich zur Verfügung.

5. Wenn die Behandlung eines Patienten in einem Funktionsbereich begonnen wird, dann wird diese Aktion ohne Unterbrechung durchgeführt. Vorzeitige Beendigungen von Arbeitsgängen sind nicht möglich.

6. Die Behandlungszeiten der einzelnen Patienten sind unabhängig von der Reihenfolge, in welcher die entsprechenden Aufträge von einem Funktionsbereich bearbeitet werden.

7. Die Patienten treffen zeitlich kontinuierlich im Krankenhaus ein, wobei der Ankunftszeitpunkt und die durchzuführenden Behandlungen stochastische Größen sind.

8. Es existieren Notfälle, die unmittelbar behandelt werden müssen. 
Aus den Modellannahmen resultiert, daß es sich bei der Planung und Steuerung von Krankenhausprozessen um ein dynamisches, stochastisches, mehrstufiges Problem handelt.

\subsection{Architektur von MedPaCo}

Die Patienten im Krankenhaus verfolgen - abgesehen von der Qualität der medizinischen Leistung, die hier nicht weiter thematisiert werden soll ${ }^{1}-$ das Ziel einer möglichst kurzen Durchlaufzeit. Geht man von knappen Ressourcen der Funktionsbereiche aus - und nur dieser Fall ist ablaufplanerisch relevant - kommt es zwangsläufig zu Wartezeiten für die Patienten. Ökonomisch bedeutet dies, daß die Patienten um die knappen Ressourcen konkurrieren, wobei ein früherer Behandlungstermin einen höheren Nutzen für den Patienten stiftet, als ein späterer Termin. Gleichzeitig verfolgen jedoch die Ressourcen das Ziel einer möglichst guten Auslastung, d.h. die Vermeidung von Leerlaufzeiten. Patienten und Ressourcen können demzufolge als konkurrierende Teilnehmer in einem krankenhausinternen Markt interpretiert werden, die versuchen, ihren Nutzen zu maximieren. Betrachtet man nun das Gesamtsystem, wird der optimale Plan - in Anlehnung an Pareto - genau dann erreicht, wenn sich kein Marktteilnehmer mehr verbessern kann, ohne daß sich ein anderer Marktteilnehmer verschlechtert (vgl. [Wei94, S. 87 f.]).

Überträgt man diese Überlegungen auf ein Multiagentensystem, müssen die Teilnehmer in diesem internen Markt, d.h. die Funktionsbereiche und die Patienten, als konkurrierende Agenten modelliert werden, die versuchen, ihren Nutzen durch Verhandlung mit anderen Agenten $\mathrm{zu}$ maximieren. $\mathrm{Zu}$ diesem Zweck müssen für die Agenten Zielfunktionen erstellt werden, die es ihnen erlauben, sowohl ihre derzeitige Situation als auch die Kosten bzw. den Nutzen einer Planänderung kardinal zu ermitteln. Aufgrund des dezentralen Charakters dieses Modells benötigen die jeweiligen Agenten nur Kenntnis über den Terminplan und die Zielfunktion der betreffenden Patienten bzw. Ressourcen. Ablaufrestriktionen werden hierbei als Nebenbedingungen der Zielfunktionen modelliert. Durch diese Struktur des Multiagentensystems ist es möglich, jedem Patienten- und Ressourcenagenten eine eigene Zielfunktion mit individuellen Präferenzen auf den Weg zu geben.

Im folgenden soll näher auf die Patienten- und Ressourcenagenten eingegangen werden.

\footnotetext{
${ }^{1}$ Die medizinische Ergebnisqualität liegt zum einen in den Händen des behandelnden Arztes und wird zum anderen von den Patienten meist als Hygienefaktor angesehen.
} 


\subsubsection{Patientenagenten}

Das Ziel eines Patientenagenten besteht in der Minimierung der Durchlaufzeit für seinen Patienten. Jedem realen Patienten im Krankenhaus wird ein solcher Patientenagent zugeordnet, der versucht die Ziele seines Patienten gegenüber den Zielen der Ressourcen und den anderen Patienten im Krankenhaus möglichst gut durchzusetzen (vgl. [Wei94, S. 85]).

Hierbei sieht der Patientenagent lediglich den Zeitplan (schedule) für seinen Patienten. Dies soll in der folgenden Abbildung anhand von zwei Patientenagenten (A, B) verdeutlicht werden.

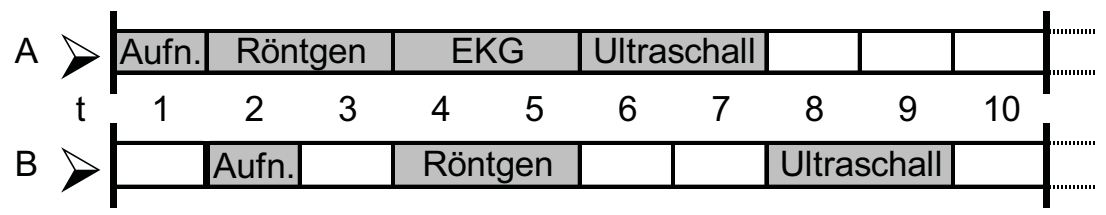

\section{Abbildung 1: Sicht der Patientenagenten}

Jeder Patientenagent verfügt über eine eigene Zielfunktion in Form einer Kostenfunktion, die es ihm erlaubt, seinen aktuellen Plan in Bezug auf das verfolgte Ziel der Minimierung der Durchlaufzeit für seinen Patienten zu bewerten und die durch eine mögliche Änderung des Zeitplans verursachten Mehr- oder Minderkosten zu ermitteln. Dieser Kostenfunktion liegt die Annahme zugrunde, daß eine Behandlung für den Patientenagenten um so teurer wird, je später sie eingeplant wird.

Neben der Kostenfunktion muß der Patientenagent die Reihenfolgerestriktionen der Behandlungen in Form von Nebenbedingungen kennen, um gültige Behandlungsreihenfolgen gewährleisten zu können. Hierbei dürfen patientengebundene Behandlungen nur sequentiell erfolgen. Für patientenungebundene Leistungen gilt diese Restriktion hingegen nicht, da sie gleichzeitig mit anderen Aktivitäten für den denselben Patienten ablaufen können.

Die gewählte Kostenfunktion der Patientenagenten orientiert sich an der von Bowman [Bow59, S. 623] vorgeschlagenen Zielfunktion, die exponentiell mit der Zeit wächst. Dadurch werden für die Patientenagenten Anreize geschaffen, Behandlungen so früh wie möglich einzuplanen, da sie im Zeitablauf immer teurer werden (vgl. [Latz97, S. 80 f.]). Zudem übersteigen die Kosten für die jeweils nächste Zeiteinheit die Summe der Kosten aller vorherigen Zeiteinheiten.

Daher hat die hier verwendete Kostenfunktion die Form $k(t)=g^{t}$, wobei $t$ die Zeit zwischen dem Ankunftszeitpunkt des Patienten im Krankenhaus und dem geplanten Anfangszeitpunkt der jeweiligen Behandlung angibt und $g$ die Priorität (Gewicht) des Patienten ausdrückt. 
Die minimale Gesamtbehandlungsdauer kann zwischen den einzelnen Patienten in Abhängigkeit von der Art und Anzahl der notwendigen Behandlungen voneinander abweichen. Daher muß sichergestellt werden, daß die Auswirkungen einer Planänderung bei allen Patienten gleich sind. Hierfür soll gedanklich der Anfangszeitpunkt jeder Behandlung soweit nach hinten verschoben werden, bis der Endzeitpunkt der minimalen Gesamtdurchlaufzeit bei allen Patienten gleich ist.

Als Normierung soll daher die folgende Definition gelten:

$$
t^{\text {Norm }}:=-\delta^{\text {Gesamt }}+t-1
$$

Wobei $t^{\text {Norm }}$ den normierten Startzeitpunkt einer Behandlung, $\delta^{\text {Gesamt }}$ die minimale Gesamtdurchlaufzeit und $t$ den realen Startzeitpunkt einer Behandlung ausdrückt. Im folgenden wird für $t^{\text {Norm }}$ nur noch $t$ geschrieben.

Da es sich bei Prozessen nicht um einzelne Zeitpunkte, sondern um Zeitspannen handelt, ist es notwendig, die Fläche unter der Kostenfunktion für die jeweiligen Behandlungen zu betrachten. Dementsprechend lautet die Kostenfunktion für eine bestimmte Behandlung:

$$
K(t, \delta)=\int_{t}^{t+\delta} g^{t}=\frac{1}{\ln g}\left(g^{t+\delta}-g^{t}\right),
$$

Die Gesamtkosten aller Behandlungen für einen Patientenagenten ergeben sich dementsprechend aus der Summe der Kosten für die einzelnen Behandlungen.

Betrachtet man nun die Auswirkungen einer Änderung des Zeitplans eines Agenten, entspricht die Kosteneinsparung (Mehrkosten) einer zeitlichen Verschiebung einer Behandlung nach vorne (hinten) genau der Differenz der Kosten dieser Zeitspannen. Demnach lassen sich die Mehrkosten bzw. die Kosteneinsparungen (=Nutzen) einer Planänderung für einen Patientenagenten wie folgt berechnen:

$$
p\left(t, t^{\text {neu }}, \delta\right)=\int_{t}^{t+\delta} g^{t}-\int_{t^{\text {neu }}}^{t^{\text {neu }}+\delta} g^{t^{\text {neu }}}
$$

oder nach Auflösung des Integrals

$$
p(t, \Delta, \delta)=\frac{1}{\ln g} g^{t}\left(1-g^{-\Delta}\right)\left(-1+g^{\delta}\right) ; \text { mit } \Delta=t-t^{\text {пеи }},
$$

wobei $t$ den bisherigen Startzeitpunkt einer Behandlung, $t^{\text {neu }}$ den neuen Startzeitpunkt, $\Delta$ die zeitliche Verschiebung und $\delta$ die Behandlungsdauer ausdrückt. 
Da diese Mehr- bzw. Minderkosten genau den Betrag angeben, den ein Patientenagent als Anbieter für eine zeitliche Verschlechterung mindestens fordern muß (Verkaufspreis), bzw. ein Patientenagent als Nachfrager maximal für eine Verbesserung $\mathrm{zu}$ zahlen bereit wäre (Kaufpreis) - um sich nicht nach der Veränderung schlechter zu stellen als vorher -, kann diese Funktion auch als Preisfunktion für Planänderungen bezeichnet werden.

Durch den konvexen Verlaufs der Kostenfunktion haben später eingeplante Behandlungen einen größeren Nutzen von einer Verschiebung nach vorne als früher eingeplante Blöcke. Dies entspricht auch der Forderung von Decker/Li [DeLi99, S. 14], daß eine Behandlung, die früher starten kann eine höhere Priorität haben sollte, als eine Behandlung, die erst später starten kann. Weiterhin wird durch den exponentiellen Charakter der Kostenfunktion gewährleistet, daß der Nutzen einer zeitlichen Verbesserung einer Behandlung, die Kosten einer zeitlichen Verschlechterung einer anderen Behandlung bis zu diesem Zeitpunkt, übersteigt.

\subsubsection{Ressourcenagenten}

Das Ziel der Ressourcenagenten ist eine möglichst gute Auslastung der von ihnen abgebildeten Ressource. Sie sehen - analog zu den Patientenagenten lediglich ihren Bereich, was an zwei exemplarischen Ressourcenagenten (Röntgen und EKG) verdeutlicht werden soll, wobei A, B, C für die Patienten steht.

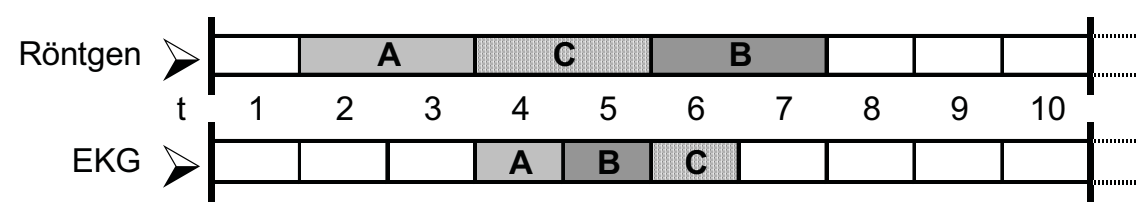

\section{Abbildung 2: Sicht der Ressourcenagenten}

Da die bei den Patientenagenten vorgestellte Kostenfunktion Wartezeiten bzw. Leerlaufzeiten durch höhere Kosten für die folgenden Behandlungen bestraft, können die Überlegungen zu den Patientenagenten auf die Ressourcenagenten übertragen werden. Daher soll den Ressourcenagenten die selbe (exponentielle) Kostenfunktion wie den Patientenagenten zugrunde gelegt werden. Ebenfalls ist hier eine Normierung notwendig, da die minimale Bearbeitungsdauer der einzelnen Funktionsbereichen unterschiedlich sein kann. Folglich ergibt sich auch für die Ressourcenagenten die Kostenfunktion

$$
K(t, \delta)=\frac{1}{\ln g}\left(g^{t+\delta}-g^{t}\right)
$$

mit 


$$
t^{\text {Norm }}:=-\delta^{\text {Gesamt }}+t-1 \text {. }
$$

Das Verhalten dieser Kostenfunktion deckt sich damit mit dem der Patientenagenten und soll hier nicht weiter dargestellt werden.

\subsection{Koordinationsmechanismus}

Nachdem in den vorherigen Abschnitten auf die Ausgestaltung der Nutzenfunktionen der jeweiligen Agenten eingegangen worden ist, soll im folgenden die konkrete Einplanung und die zur Verbesserung notwendige Verhandlung zwischen den Agenten beschrieben werden.

Der Verhandlung bzw. Koordination liegt die Idee zugrunde, daß ein Patientenagent versucht, Behandlungen zeitlich nach vorne zu verschieben. Da spätere Behandlungen teurer sind als frühere, kann er durch dieses Vorgehen Kosten sparen. Falls die gewünschte Zeitspanne bereits von einem anderen Patientenagenten belegt ist, kann er diese (potentielle) Kostenersparnis dazu verwenden, die gewünschte Zeitspanne von einem anderen Agenten abzukaufen. Da die Aufgabe der Zeitspanne für den Verkäufer in der Regel eine Verschlechterung bedeutet, wird dieser die Zeitspanne nur aufgeben, wenn seine zusätzlichen Kosten durch den Käufer ausgeglichen werden. Dies bedeutet, daß die Kostenersparnis des Käufers größer als die zusätzlichen Kosten des Verkäufers sein muß. Das bedeutet, daß der Nutzen des Käufers den Nutzenverlust des Verkäufers übersteigen muß.

Die Verhandlung läuft nach folgendem Schema ab:

1. Der nachfragende Patientenagent ermittelt im Rahmen seiner Reihenfolgerestriktionen die Behandlung/Untersuchung, die ihm durch eine Umplanung auf den frühest möglichen Termin, die größte Kostenersparnis (Nutzen) bringt. Dabei sind die Reihenfolgerestriktionen so zu interpretieren, daß eine Behandlung nicht vor bzw. gleichzeitig mit einer Behandlung des selben Patienten eingeplant werden darf, die vorher erbracht worden sein muß. Bestehen keine sequentiellen Interdependenzen, ist eine Überschneidung mit anderen Behandlungen des selben Agenten möglich. Handelt es sich bei den betroffenen Behandlungen um patientengebundene Leistungen, werden diese Behandlungen wie Anbieter interpretiert. Handelt es sich hierbei jedoch um patientenungebundene Leistungen, so werden sie nicht weiter berücksichtigt, da kein Konflikt bei gleichzeitiger Ausführung besteht.

2. Danach eröffnet der nachfragende Agent die Verhandlung um den gewünschten Zeitraum im gewünschten Funktionsbereich. Hierzu teilt er dem Ressourcenagenten dieses Funktionsbereiches seinen bisherigen und den gewünschten Zeitraum mit. 
3. Der Ressourcenagent, der für diesen Funktionsbereich zuständig ist, markiert den bisherigen Zeitraum des Nachfragers als verfügbar (im Rahmen der Verhandlung) und den gewünschten Termin als für den Nachfrager reserviert.

4. Danach informiert dieser Ressourcenagent anhand seines lokalen Plans alle tangierten Patientenagenten (Anbieter). Ist ein Funktionsbereich in der Lage, mehr als einen Patienten gleichzeitig zu behandeln, werden alle alternativen Anbieter zur Abgabe eines Angebots aufgefordert.

5. Die anbietenden Patientenagenten ermitteln daraufhin die durch die Planänderung verursachten Kosten und teilen diese dem Nachfrager mit. Da sie zur Ermittlung dieser Kosten den Preis des gewünschten Ausweichtermins kennen müssen, treten die Anbieter wiederum als Nachfrager nach diesem alternativen Termin auf. Sie versuchen sich hierbei so früh wie möglich einzuplanen - was bei den anbietenden Agenten auch ein späterer als der bisherige Termin sein kann, da sie die zusätzlichen Kosten von dem ersten Nachfrager (Initiator) ausgeglichen bekommen. Eine Einplanung auf einen reservierten Termin ist nicht möglich.

6. Ist eine zeitliche Verschiebung für einen anbietenden Agenten aufgrund eines Konfliktes mit einer Behandlung, die erst nach Abschluß der umzuplanenden Behandlung durchgeführt werden darf, nicht möglich, so muß auch diese Behandlung verschoben und die daraus resultierenden Kosten dem Nachfrager in Rechnung gestellt werden.

7. Liegt eine Überschneidung mit einer Behandlung des eigenen Patienten in einem anderen Funktionsbereich vor, so ermittelt der Nachfrager gleichzeitig als Anbieter die Kosten für die Planänderung dieser Behandlung.

8. Ist ein von den Anbietern gewünschter Ausweichtermin von einem anderen Agenten belegt, durchlaufen sie als Nachfrager ebenfalls die Schritte 2 bis 7 . Ist der gewünschte Termin frei, sind sie in der Lage, die Kosten der Umplanung direkt zu ermitteln und dem jeweiligen Nachfrager mitzuteilen. War dieser Nachfrager gleichzeitig Anbieter, so kann nun auch er seinem Nachfrager die Kosten seiner Planänderung mitteilen. Dieser Prozeß setzt sich fort, bis der erste Anbieter dem ersten Nachfrager (Initiator) seine Kosten mitteilen kann. Der Preis des ersten Anbieters umfaßt damit die Kosten aller von der Umplanung tangierten Patientenagenten.

9. Der Initiator vergleicht nun seine potentielle Kostenersparnis (=Nutzen) aus der Umplanung mit dem Preis des Anbieters und übermittelt die Bestätigung des Tauschvorganges, wenn sein Nutzen die Kosten übersteigt. In diesem Falle werden alle reservierten Termine von den entsprechenden Agenten belegt und die bisherigen Termine endgültig freigegeben. Falls seine Kostenersparnis kleiner als der Preis des Anbieters ist, werden die Reservierungen gelöscht und die alten Termine beibehalten. Falls der betroffene Funktionsbereich in der Lage ist, zu dem gewünschten Termin mehrere Patienten zu behandeln, wird 
nur das Angebot des kostengünstigsten Agenten bei der Kostenkalkulation berücksichtigt.

Da die zusätzlichen Kosten der Anbieter in dem vorgestellten Koordinationsmechanismus durch die Kostenersparnis des ersten Nachfragers (Initiator) ausgeglichen werden, können sie sich auch auf Zeitspannen einplanen, bei denen sie Verlust machen, d.h. ihre Kostenersparnis kleiner als der Preis für diesen Zeitraum ist. Aufgrund dieser Tatsache kann das hier vorgestellte Einplanungsverfahren, nach den bisherigen Erkenntnissen der Konzeptphase, nicht in einem lokalen Optimum bzw. Suboptimum stecken bleiben, da jeder Tausch prinzipiell möglich ist, solange sich der Gesamtplan verbessern kann.

Im weiteren ist der Koordinationsmechanismus in der Lage, ad hoc auf Änderungen einzugehen. Verschlechtert sich beispielsweise der Gesundheitszustand eines Patienten besteht die Möglichkeit, daß er seine Priorität (g) erhöht und somit einen Vorteil bei der Verhandlung erhält. Im Falle zusätzlich notwendig werdender Behandlungen werden diese kostenlos in die nächste konfliktfreie Zeitspanne eingeplant. Kommt es zu einem temporären Ausfall einer Ressource, werden die betroffenen Untersuchungen zunächst analog $\mathrm{zu}$ den neuen Behandlungen kostenlos auf den nächsten freien Platz eingeplant. Von dieser Stelle aus können sie sich durch Verhandlungen wieder verbessern.

\subsection{Beispiel}

Im folgenden soll der Einplanungsmechanismus an einem einfachen Beispiel illustriert werden. Zur besseren Übersichtlichkeit wird lediglich die Sicht der Funktionsbereiche dargestellt.

Abbildung 3 zeigt eine Ausgangssituation mit zwei Funktionsbereichen (FB1, FB2) und drei Patienten (A, B, C). Als weitere Information werden die Kosten der Patientenagenten für die jeweilige Zeitspanne angegeben. Hierbei werden die Kosten der Funktionsbereiche $\mathrm{zu}$ den (internen) Kosten der Patientenagenten addiert. In diesem Beispiel kann ein Patient nur in einem Funktionsbereich gleichzeitig behandelt werden, wobei die Reihenfolge dieser Behandlungen beliebig ist. Weiterhin wird die Priorität (g) aller Agenten auf "2" gesetzt.

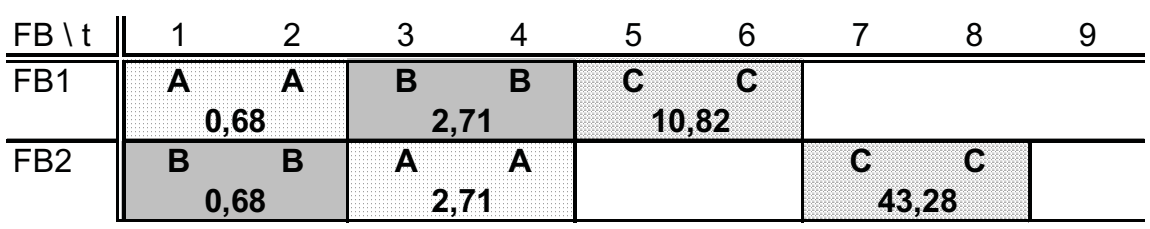

\section{Abbildung 3: Patientenagenten vor Umplanung}

Der Patientenagent $C$ kann nun versuchen, sich durch eine Verhandlung mit dem Ressourcenagenten B im Funktionsbereich 2 zu verbessern. Zur Ermittlung seines 
Verkaufpreises muß nun der Patientenagent B die Kosten für eine Umplanung auf die Zeitspanne [3,4] ermitteln. Dafür tritt er als Nachfrager und der Patientenagent A als Anbieter dieser Zeitspanne im Funktionsbereich 2 auf. Gleichzeitig muß B nun auch die Rolle des Anbieters im Funktionsbereich 1 übernehmen, da zwei Behandlungen von B nicht gleichzeitig ablaufen dürfen. Analog zu dem vorherigen Vorgehen fragt nun A die Zeitspanne [5,6] im Funktionsbereich 2 und B die Zeitspanne [1,2] im Funktionsbereich 1 nach, was dazu führt, daß A nun auch im Funktionsbereich 1 die Zeitspanne [3,4] nachfragen muß. Da sich A in den letzten Schritten auf freie Zeitspannen einplanen konnte, kann nun A die Kosten der Umplanung für die Funktionsbereiche $1(2,71-0,68=2,03)$ und 2 $(10,82-2,71=8,11)$ an B weitergeben. Daraufhin kann B seine Kosten im Funktionsbereich $1 \quad(0,68-2,71+2,03=0)$ ermitteln und sich selbst für die Umplanung im Funktionsbereich 2 in Rechnung stellen. Da nun B für den Funktionsbereich 2 die Preise von B im Funktionsbereich 1 (0) und A im Funktionsbereich $2(8,11)$ kennt, kann B seine Kosten der Umplanung (2,71$0,68+8,11=10,14)$ an $\mathrm{C}$ übermitteln. Der Patientenagent $\mathrm{C}$ wird diesen Tausch annehmen, da seine Kostenersparnis $(43,28-0,68=42,6)$ den Preis für diese Zeitspanne $(10,14)$ übersteigt. Abbildung 4 zeigt das Ergebnis mit den neuen Kosten.

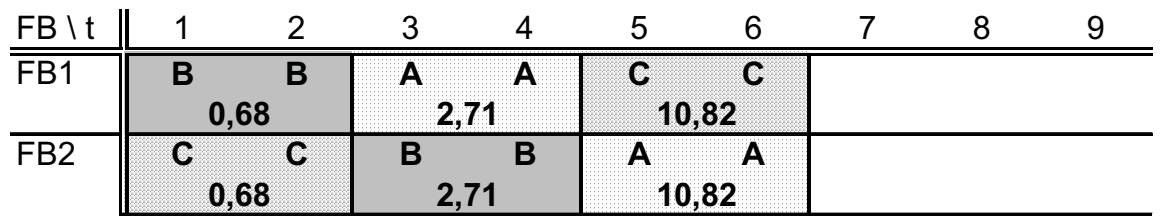

\section{Abbildung 4: Patientenagenten nach Umplanung}

\subsection{Erste Würdigung}

Durch die vorgestellte exponentielle Ziel- bzw. Kostenfunktion werden für die Patientenagenten Anreize zu einer möglichst frühen Einplanung und für die Ressourcenagenten Anreize zu einer Vermeidung von Leerlaufzeiten, geschaffen. Da diese - zu minimierende - Zielfunktion implizit Prioritäten für die jeweiligen Behandlungen der einzelnen Patienten bestimmt, ist eine genauere Steuerung als durch feste Maluspunkte für Wartezeiten bzw. Verspätungen möglich (vgl. [Wei94]). Weiterhin ermöglicht die Zielfunktion eine explizite Gewichtung der einzelnen Patienten bzw. Funktionsbereiche, wodurch beispielsweise der Gesundheitszustand eines Patienten berücksichtigt werden kann.

Zwar ist es prinzipiell möglich das hier gewählte Einplanungsverfahren auch rein zentral zu modellieren, jedoch weist die hier gewählte dezentrale Planabstimmung Vorteile auf, da die Information in Krankenhäusern auf die einzelnen Funktionsbereiche verteilt ist. Bei einer zentralen Koordination müßte jede 
Änderung in einem Funktionsbereich oder bei einem Patienten an den zentralen Planer übermittelt werden, aufgrund derer ein neuer Gesamtplan erstellt werden müßte [Adam93, S. 470 ff.]. Bei einem dezentralen Koordinationsmechanismus werden jedoch nur die von einer Änderung betroffenen Pläne durch gezielte Verhandlungen geändert. Dadurch bleibt das System auch bei sehr hoher Komplexität flexibel und funktionsfähig. Weiterhin ist das Einplanungsverfahren durch dezentrale Koordination beliebig skalierbar, d.h. zusätzliche Patienten oder Funktionsbereiche können im Gegensatz zu einer zentralen Lösung problemlos berücksichtigt werden (vgl. hierzu auch [HaGe99, S. 4 f.]).

Dieser Sachverhalt ist auch aus der Marktwirtschaft-Planwirtschaft-Kontroverse bekannt, da auch hier die nötigen Informationen über Nutzenvorstellungen und Interdependenzen für eine funktionsfähige zentrale Planung fehlen. Ebenso kann auch eine zentrale Planwirtschaft nicht unmittelbar auf unvorhergesehene Änderungen reagieren.

\subsection{Offene Probleme}

Bisher wurde als Ziel der Patienten zunächst nur die Minimierung der Durchlaufzeit thematisiert und hierfür eine Kostenfunktion modelliert. Daneben verfolgen jedoch stationäre Patienten auch das Ziel einer zeitlichen Zusammenlegung von Behandlungen, da sie bei einem zeitlichen Auseinanderfallen der Behandlungen zwischen diesen Behandlungen zurück auf ihre Station müssen, was zu zusätzlichen Wegen und Transportaufwand führt. Dieses Ziel könnte beispielsweise als Kostenfunktion in der Form einer Sprungfunktion modelliert werden, bei der keine Kosten anfallen, solange die Behandlungen geblockt durchgeführt werden und sonst ein fester Betrag für jede Unterbrechung berechnet wird.

\section{Zusammenfassung und Ausblick}

In dem vorliegenden Beitrag wurde das Problem der Patientensteuerung im Krankenhaus beschrieben und eine eigene Konzeption eines Koordinationsmechanismus zur dezentralen Ablaufplanung in medizinischen Behandlungspfaden dargestellt, der auf dem Prinzip der Koordination in der Marktwirtschaft basiert. Dieser Koordinationsmechanismus soll zukünftig in ein Multiagentensystem eingebettet werden, in dem die Patienten und Funktionsbereiche (Ressourcen) als Agenten mit eigenen Zielen agieren. Hierfür wurde eine Zielfunktion in der Form einer Kostenfunktion für Patienten- und Ressourcenagenten modelliert, die es den jeweiligen Agenten erlaubt, sowohl die Kosten ihres aktuellen Schedules als auch die Mehr- bzw. Minderkosten einer Planänderung zu bewerten. 
Im Rahmen der ersten Würdigung von MedPaCo wurde gezeigt, daß ein dezentraler Ansatz zur Patientensteuerung aufgrund der dezentralen Informationsverteilung im Krankenhaus und der besseren Flexibilität und Skalierbarkeit besser geeignet erscheint, als ein rein zentrales Verfahren.

Weiterer Forschungsbedarf besteht in der Lösung des Problems der Zielsetzung des zeitlichen Zusammenfallens von Behandlungen, $\mathrm{zu}$ denen derzeit nur eine erste Idee präsentiert werden konnte. Auch gilt es die hier vorgestellte Konzeption eines Koordinationsverfahrens $\mathrm{zu}$ realisieren und gegen zentrale Einplanungsverfahren zu bewerten.

\section{Literaturverzeichnis}

[Adam93] Adam, D.: Produktions-Management, 7. Aufl., Gabler, Wiesbaden 1993.

[Adam96] Adam, D.: Planung und Entscheidung: Modelle-Ziele-Methoden, 4. Aufl., Gabler, Wiesbaden 1996.

[Bow59] Bowman, E. H.: The schedule-sequencing problem, in: Operations Research, (1959) 7, S. 621-624.

[DeLi98] Decker, K.; Li, J.: Coordinated Hospital Patient Scheduling, in: Proceedings of the Third International Conference on Multi-Agent Systems, Paris, 7/98.

[DeLi99] Decker, K.; Li, J.: Coordinating Mutually Exclusive Resources using GPGP, in: Autonomous Agents and Multi-Agent Systems, 3 (1999) 2, Kluwer Academic publishers, Boston

[Gäf90] Gäfgen, G.: Gesundheitsökonomie, Nomos-Verl.-Ges., Baden-Baden 1990.

[Gie76] Gierl, L.: Partialmodelle der Ablaufsteuerung im Krankenhaus auf empirischer Grundlage, Diss., Uni Erlangen - Nürnberg 1976.

[HaGe99] Hannebauer, M.; Geske, U.: Coordinating Distributed CLP-Solvers in Medical Appointment Scheduling, Berlin 1999.

[Her94] Herder-Dorneich, P.: Ökonomische Theorie des Gesundheitswesens: Problemgeschichte, Problembereiche, Theoretische Grundlagen, Nomos-Verl.-Ges., Baden-Baden 1994.

[Latz97] Latz, Th.: Entscheidungsmodelle der Ablaufplanung, Diss., Dt. Univ.Verl., Wiesbaden 1997.

[Sch190] Schlüchtermann, J.: Patientensteuerung: am Beispiel der Radiologie eines Krankenhauses, Diss., Josef Eul Verlag GmbH, Bergisch Gladbach 1990. 
[SchmUr99] Schmidt, B.; Urban, C.: Operative Planung und Steuerung von Gesundheitsdiestleistungen im Krankenhaus: Der Einsatz von PECSAgenten im medizinischen Bereich, internes Arbeitspapier, Universität Passau, Passau 1999.

[Wei94] Weigelt, M.: Dezentrale Produktionssteuerung mit Agenten-Systemen: Entwicklung neuer Verfahren und Vergleich mit zentraler Lenkung, Dt. Univ.-Verl., Wiesbaden 1994.

[Wen87] Wendt, K. L. v.: Patientensteuerung an Computer-Tomographen, Dipl. Arb., in: Veröffentlichungen des Instituts für Industrie- und Krankenhausbetriebslehre der Westfälischen Wilhelms-Universität Münster, Universität Münster, Münster 1987. 
In der vorliegende Arbeit wird eine Konzeption für ein Koordinationsverfahren zur dezentralen Ablaufplanung in medizinischen Behandlungspfaden (MedPaCo) entwickelt. Hierzu werden die ablaufplanerisch relevanten Eigenschaften von Krankenhausprozessen analysiert und ein Überblick über die wesentlichen Beiträge zur dezentralen Planung in Krankenhäusern gegeben. Im Anschluß daran wird die Architektur von MedPaCo beschrieben. Basierend auf einem Multiagentensystem greift MedPaCo das Konzept der marktwirtschaftlichen Koordination auf. Hierbei werden die Patienten und Funktionsbereiche im Krankenhaus durch Patienten- und Ressourcenagenten repräsentiert, die über eigene Ziele und Restriktionen verfügen. Danach wird der Koordinationsablauf dargestellt und an einem Beispiel illustriert. Zum Schluß wird eine erste Würdigung von MedPaCo vorgenommen und auf offene Probleme eingegangen. 